\title{
Violencia doméstica y riesgo de conducta suicida en universitarios adolescentes
}

\author{
Francisco Espinoza-Gómez, D en C, (I) Víctor Zepeda-Pamplona, M en SP,(I) \\ Víctor Bautista-Hernández, D en C, (I) Carlos Moisés Hernández-Suárez, $\mathrm{PhD}$,(I) \\ Oscar Alberto Newton-Sánchez, D en C, (1) Guadalupe R Plasencia-García. ${ }^{(2)}$
}

\author{
Espinoza-Gomez F, Zepeda-Pamplona V, \\ Bautista-Hernández V, Hernández-Suárez $C M$, \\ Newton-Sánchez OA, Plasencia-García GR. \\ Violencia doméstica y riesgo de conducta suicida \\ en universitarios adolescentes. \\ Salud Publica Mex 2010;52:213-219.
}

\section{Resumen}

Objetivo. Estimar el grado de asociación entre violencia doméstica física, verbal y sexual con la conducta suicida en adolescentes universitarios. Material y métodos. Estudio de casos y controles pareado en estudiantes universitarios de Colima. Los casos fueron 235 adolescentes que presentaron tanto ideación suicida como intento suicida; los controles fueron 470 individuos de la misma edad y sexo. Resultados. El abuso sexual mostró el mayor grado de asociación con conducta suicida $(R M=27.4)$, seguido de violencia verbal $(R M=$ 9.28), uso de drogas $(R M=8.6)$, violencia física $(R M=5.5)$ y tabaquismo $(R M=3.6)$. La regresión logística multivariada mostró que la violencia verbal se asoció con conducta suicida en forma independiente, mientras que violencia física, abuso sexual, tabaquismo y uso de drogas parecen depender de aquella. Conclusiones. La violencia intrafamiliar, particularmente la verbal, está fuertemente asociada con la conducta suicida en adolescentes y debe ser considerada dentro de programas preventivos contra suicidio.

Palabras clave: suicidio; violencia doméstica; adolescente; México
Espinoza-Gomez F, Zepeda-Pamplona V, Bautista-Hernández V, Hernández-Suárez $\mathrm{CM}$, Newton-Sánchez OA, Plasencia-García GR. Domestic violence and risk of suicidal behavior among university students.

Salud Publica Mex 2010;52:213-219.

\begin{abstract}
Objective. To estimate the degree of association between domestic violence - physical, verbal or sexual- with suicidal behavior among university students. Material and Methods. A matched case-control study was done with students attending the University of Colima, Mexico. The cases were 235 teenagers who presented both suicidal ideation and suicide attempt; the controls were 470 individuals of the same age and sex. Results. Sexual abuse showed the highest degree of association with suicidal behavior $(O R=27.4)$, followed by verbal violence $(O R=9.28)$, drug use $(O R=8.6)$, physical violence $(O R=5.5)$ and smoking $(O R=3.6)$. Multivariate logistic regression showed that verbal violence was associated with suicidal behavior independently of the other variables, while physical violence, sexual abuse, smoking and drug use seem to depend on verbal violence. Conclusions. Domestic violence, particularly verbal or sexual, is strongly associated with suicidal behavior in adolescents and should be considered in suicide prevention programs.
\end{abstract}

Key words: suicide; domestic violence; adolescent; Mexico.

(I) Facultad de Medicina, Universidad de Colima. Colima, México .

(2) Facultad de Psicología, Universidad de Colima. Colima, México.

Fecha de recibido: 28 de mayo de 2009 - Fecha de aceptado: 13 de enero de 2010 Solicitud de sobretiros: Dr. Francisco Espinoza- Gómez. Laboratorio de Salud Pública, Facultad de Medicina, Universidad de Colima. Av. Universidad 333, col. Las Víboras. 28040, Colima, México.

Correo electrónico: fespin@cgic.ucol.mx 
E suicidio es un grave problema de salud pública en el ámbito mundial, particularmente cuando se presenta en adolescentes y jóvenes, pues este dramático evento eleva notablemente la carga social y económica de las sociedades afectadas. ${ }^{1} \mathrm{El}$ incremento observado en las últimas décadas en la frecuencia de suicidio entre grupos de edad cada vez menores ${ }^{2}$ ha alertado a las autoridades sanitarias a nivel mundial para la búsqueda de estrategias que permitan la detección oportuna de factores de riesgo y así implementar programas preventivos más eficientes. ${ }^{3}$ A pesar de esta importante demanda, la identificación de factores que pudieran ser predictores de suicidio ha sido desalentadora, dada la naturaleza multidimensional del acto suicida. ${ }^{4-6}$ Los factores que con mayor fuerza se han asociado al suicidio en adolescentes han sido la presencia de enfermedad psiquiátrica subyacente, la historia familiar de suicidio ${ }^{6,7} \mathrm{y}$, en menor medida, la conducta impulsiva, el abuso de alcohol, de tabaco y otras substancias psicoactivas. ${ }^{8,9}$

Por otro lado, la ideación suicida y el intento suicida derivan en la consumación de suicidio en hasta $10 \%$ de los casos, ${ }^{10,11}$ es por ello que la identificación de estas conductas, sobre todo cuando ambas coinciden en un individuo, debe ser considerada como una importante señal de alarma respecto a la consumación de suicidio. En este sentido, el reconocimiento de los factores de riesgo asociados a la ideación y al intento suicida podría contribuir al diseño de programas preventivos más eficientes y oportunos. ${ }^{2,4}$ La ideación suicida y el intento suicida en adolescentes tienen orígenes multifactoriales dentro de los que destacan aspectos biológicos como el sexo femenino, la edad entre 12 y 15 años, así como factores ambientales y sociales tales como nivel socioeconómico bajo, el fracaso escolar y la desintegración familiar. ${ }^{12-14}$ La violencia doméstica, ya sea física o verbal, podría ser también un condicionante de conductas autodestructivas, incluyendo la suicida. Por ejemplo, se ha encontrado una fuerte asociación entre abuso sexual en la infancia e ideación suicida, ${ }^{15}$ mientras que otros autores han descrito fuerte relación entre violencia escolar, discriminación y agresiones físicas con el desarrollo de baja autoestima y conducta suicida. ${ }^{5,16}$ El trato violento hacia los adolescentes, particularmente aquél dirigido contra las mujeres, parece ser mucho más común de lo pensado y ser causal importante de trastornos emocionales en grandes sectores de la población. ${ }^{17}$ En México recientemente se encontró asociación entre suicidio infantil y violencia familiar por medio de un estudio retrospectivo en el estado de Guanajuato, ${ }^{18}$ mientras que en otro estudio se reporta fuerte asociación entre violencia sexual e intento suicida en mujeres. ${ }^{19}$ Sin embargo, existe muy poca información en nuestro medio respecto a la asociación entre violencia y conducta suicida en adolescentes y menos aún, estudios que ponderen el grado de asociación específica entre la violencia doméstica y la conducta suicida en comparación con los factores tradicionalmente reconocidos y arriba señalados.

Con el objeto de analizar la participación de la violencia doméstica, ya sea verbal, física o sexual, como un factor de riesgo específico para la aparición de conducta suicida en adolescentes y su posible interacción con otras variables, se llevó a cabo el presente estudio de casos y controles entre alumnos que ingresan a la Universidad de Colima, México, a través de una encuesta que se realiza dentro del programa denominado "Universidades saludables de México".

\section{Material y métodos}

La población de estudio comprende a los estudiantes que ingresan a la Universidad de Colima, México. El estado de Colima cuenta con 567996 habitantes, de los cuales aproximadamente 56000 son adolescentes (INEGI). ${ }^{20}$ De éstos, los estudiantes de la Universidad de Colima constituyen aproximadamente 38\% (21 166 estudiantes). A todos los alumnos que ingresan a la Universidad se les aplica una encuesta sobre tópicos de salud denominada Examen Médico Automatizado (EMA) que consta de 63 reactivos de autorrespuesta que exploran aspectos relativos al historial médico, familiar, social y económico, así como la presencia de adicciones, hábitos y condiciones psicológicas de los estudiantes. El EMA ha sido diseñado y validado por la Universidad Nacional Autónoma de México. ${ }^{21}$

\section{Selección de casos y controles}

Dentro del EMA existen reactivos que permiten identificar la presencia de ideación suicida y de intento suicida en los últimos 5 años. A partir de los datos de la encuesta, que se aplicó a 5926 alumnos que ingresaron a la Universidad de Colima durante julio de 2006, se seleccionaron 235 casos de personas entre los 14 y 19 años, quienes refirieron datos francos de ideación suicida aunado a intento suicida, así como un grupo de 470 controles del mismo sexo y edad de los casos, que fueron seleccionados en forma aleatoria entre estudiantes que negaron haber tenido ideación o intento suicida en la misma encuesta. El número de casos se calculó tomando como base una frecuencia de violencia familiar en México de aproximadamente $33 \% 22$ y una razón de momios de 1.9 en relación con los casos, considerando un promedio de la fuerza de asociación entre violencia sexual e intento suicida reportada por otros autores, que va de 1.3 a 4.3..$^{15,19} \mathrm{El}$ apareamiento 
de controles se hizo 2 a 1, considerando mismo sexo y edad de los controles.

\section{Definición de variables}

La presencia de ideación suicida se definió de manera nominal dicotómica con base en la respuesta positiva a las siguientes preguntas del EMA: En el último mes, 1) ¿Has sentido que no vale la pena vivir?; 2) ¿Has deseado dejar de existir? y 3) ¿Has pensado que sería mejor morir que vivir? Se consideró positiva cuando al menos dos respuestas fueron positivas. El índice de concordancia de Chronbach para estas preguntas resultó de 0.79 .

El intento suicida igualmente se registró de manera dicotómica ( $(s i ́=1$, no $=0)$, con base en la respuesta a la pregunta: En los últimos 5 años, ¿has intentado alguna vez quitarte la vida? Los casos son estudiantes que contestaron positivamente a ambas preguntas y los controles refirieron respuesta negativa en ambas.

La percepción de violencia doméstica se dividió en las dimensiones: a) violencia física, cuando se refirió maltrato en casa a base de golpes o lesiones, a través de la pregunta ¿alguna vez te han agredido fuertemente con golpes en tu casa?; b) violencia verbal: cuando el alumno contestó positivamente a la pregunta: ¿alguna vez te han agredido fuertemente con insultos y humillaciones en casa? y c) abuso sexual, en el caso de responder afirmativamente a la pregunta: ¿te han violado o abusado sexualmente en tu casa? Estas variables se codificaron en forma dicotómica (sí =1, no =0). En ningún caso se señaló al agresor. Estas preguntas fueron diseñadas por la Dirección General de Salud Mental de la UNAM como parte del EMA. ${ }^{21}$

Otras variables exploradas como posibles factores de confusión fueron las siguientes:

a) Tabaquismo positivo, cuando el consumo de cigarrillos fue igual o mayor a uno diario $(=1)$ y negativo $(=0)$ cuando el consumo fue menor a uno por semana, con base en la definición propuesta por el estudio MONICA de la OMS para el riesgo cardiovascular. ${ }^{23}$

b) La presencia de alcoholismo, que se clasificó de acuerdo con los criterios de la OMS y el programa AUDIT: ${ }^{24}$ positivo $(=1)$ cuando el consumo semanal fue igual o mayor a 6 copas de licor con $35^{\circ}$ de alcohol y negativo $(=0)$ si el consumo reportado fue menor a esa cifra.

c) El consumo regular de drogas psicotrópicas (amfetaminas, cocaína o sedantes) también se codificó en forma dicotómica $(s i ́=1$, no $=0)$. d) El nivel escolar se clasificó como: bachillerato $(=1)$ y profesional $(=0)$.

e) Nivel socioeconómico, que se manejó como bajo $(=1)$, cuando el alumno reportó carecer de vivienda propia y de enseres domésticos básicos tales como estufa de gas, refrigerador así como acceso a servicios médicos y de urbanización (luz y agua entubada), o como medio-alto $(=0)$ cuando se reportó acceso a cualquiera de los utensilios y servicios arriba señalados. El grupo de alto nivel socioeconómico se incluyó en esta última categoría dado su pequeño número.

Aspectos éticos

La aplicación de la EMA cuenta con la aprobación del Consejo General Universitario, así como con el consentimiento de los padres de familia y de los propios alumnos a través de documento firmado. Tanto el consentimiento como las hojas de resultados se mantienen bajo estricto resguardo de confidencialidad en el Departamento de Servicios Médicos de la Universidad de Colima. La utilización de dicha información para el trabajo fue aprobada por el Comité de Investigación y Bioética de la Facultad de Medicina de la Universidad de Colima.

\section{Análisis estadístico}

Todas las variables se expresan como proporciones, con sus respectivos intervalos de confianza a 95\% (IC 95\%). Para explorar la asociación entre las variables dicotómicas se utilizaron tablas de contingencia con cálculo de $\chi^{2}$ y razón de momios $(R M)$ de Mantell-Haenzell para casos y controles pareados, así como sus respectivos IC 95\% por el método de Cornfield. Con el fin de explorar la posible interacción entre la conducta suicida con los otros factores de confusión explorados en la EMA, se utilizó regresión logística multivariada no condicionada con análisis de los productos de interacción, de acuerdo con las recomendaciones de Katz. ${ }^{25}$ Para ello se utilizaron los programas EPIDAT 3.1 (OPS) y Logistic Regression. ${ }^{26}$ Las asociaciones estadísticamente significativas se definieron cuando los IC 95\% de las RM no cruzaran el valor de 1 y el valor $p$ fuera $\leq 0.01$.

\section{Resultados}

De los 5926 estudiantes que completaron la encuesta, 5484 fueron adolescentes entre 14 y 19 años: 2934 mujeres y 2550 varones, con una edad promedio de 16 años (IC 95\%: 15.4-16.4), distribuidos de la siguiente manera: 2801 entre 14 y 15 años; 1185 entre 16 y 17 años; 
1598 entre 18 y 19 años. Respecto al nivel educativo: 3701 estudiantes pertenecen al nivel medio superior (bachillerato) y 1783 al nivel superior o profesional. El nivel socioeconómico por grupo fue: bajo, en 853 y medio-alto en 4631 alumnos.

En el cuadro I se señala la frecuencia de respuestas relacionadas con ideación suicida, intento suicida, conducta suicida (combinación de las anteriores), tabaquismo, alcoholismo, consumo de drogas, violencia familiar física, verbal y abuso sexual de manera comparativa entre hombres y mujeres en el total de estos 5484 estudiantes. Como puede apreciarse en ese cuadro, el número total de adolescentes que refirieron ideación suicida fue de $870(15.8 \%)$, mientras que 398 consignaron intento suicida (7.3\%). En total, 411 refirieron haber sido sometidos a violencia física en casa $(7.5 \%)$; 905 señalaron violencia verbal $(16.5 \%)$ y sólo $90(1.1 \%)$ refirió haber sido sometido a abuso sexual. La frecuencia de tabaquismo resultó positiva en 426 estudiantes (7.7\%); el uso de drogas en 127 de 5429 que contestaron $(2.3 \%$ ) y el alcoholismo en 505 de 2335 que contestaron (21.6\%). Se resalta que la frecuencia de ideación suicida, intento suicida y la percepción de violencia en todas sus formas resultaron sensiblemente mayores en mujeres que en hombres, mientras que el tabaquismo, el uso de drogas $\mathrm{y}$ alcohol fue menor en mujeres.

A partir de este grupo de 5484 alumnos se seleccionaron los casos entre aquellos con conducta suicida, así como sus controles. En el cuadro II se muestran las características de los casos y controles en cuanto a violencia doméstica, nivel escolar, nivel socioeconómico, tabaquismo y uso de drogas, con sus respectivos valores RM e IC 95\%. En este análisis se excluyó la presencia de alcoholismo debido al escaso número de individuos que refirieron el dato.

Con el fin de identificar posibles interacciones entre las variables más influyentes y las de confusión, se realizó análisis por medio de regresión logística multivariada. Los resultados del modelo con las cinco variables seleccionadas después del proceso de eliminación retrógrada en 702 individuos que contestaron todas las preguntas, se muestra en el cuadro III. Se resalta la persistentemente elevada tasa de $R M$ entre conducta suicida y violencia verbal, mientras que el nivel de asociación con las otras variables se redujo sustancialmente en el modelo multivariado.

Con el fin de explorar la posible interacción entre las variables más influyentes, se realizaron análisis bivariados cruzados con su respectivo producto de interacción de acuerdo con Katz. ${ }^{25}$ Dicho análisis de interacción entre violencia física, violencia verbal y conducta suicida mostró una RM de 0.61 (IC 95\%: 0.17 2.14), mientras que la interacción entre violencia verbal y tabaquismo con conducta suicida mostró una $R M$ de 1.14 (IC 95\%: 0.54-2.4). En estos análisis se excluyó el abuso sexual, a pesar de su fuerte influencia, dado que sólo se observó un caso positivo en el grupo control, que amplió inaceptablemente los IC a 95 por ciento.

\section{Cuadro I}

\section{FreCUENCIA DE IDEACIÓN SUICIDA E INTENTO SUICIDA, VIOLENCIA FísICA, VIOLENCIA VERBAL, ABUSO SEXUAL, TABAQUISMO,

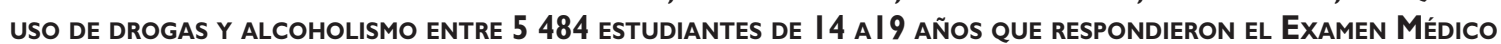 Automatizado (EMA). Colima, México, julio de 2006}

\begin{tabular}{|c|c|c|c|c|}
\hline Variable & $\begin{array}{c}\text { Sexo femenino } \\
n=2934\end{array}$ & $\begin{array}{c}\text { Sexo masculino } \\
n=2550\end{array}$ & $R M$ & (IC a 95\%) \\
\hline Ideación suicida & 592 & 278 & 2.06 & $(1.77-2.4)$ \\
\hline Intento suicida & 289 & 111 & 2.4 & $(1.91-3.0)$ \\
\hline $\begin{array}{l}\text { Conducta suicida } \\
\text { (ideación+ intento) }\end{array}$ & 179 & 56 & 2.89 & $(2.13-3.9)$ \\
\hline Violencia física & 243 & 168 & 1.27 & $(1.04-1.56)$ \\
\hline Violencia verbal & 577 & 328 & 1.65 & $(1.43-1.92)$ \\
\hline Abuso sexual & 47 & 13 & 3.17 & $(1.72-5.82)$ \\
\hline Tabaquismo* & 198 & 228 & 0.73 & $(0.6-0.89)$ \\
\hline Uso de drogas ${ }^{\ddagger}$ & 38 & 89 & 0.36 & $(0.24-0.53)$ \\
\hline Alcoholismo§ & 216 & 289 & 0.62 & $(0.5 \mid-0.74)$ \\
\hline \multicolumn{5}{|c|}{$\begin{array}{l}\text { * El sexo femenino mostró un efecto "protector" sobre tabaquismo ( } p=0.003) \\
\text { ‡ El uso de drogas se consignó significativamente menos en mujeres que en varones, entre } 5429 \text { estudiantes que respondieron }(p=0.002) \\
\text { § Solamente } 2335 \text { individuos contestaron en relación con alcoholismo }\end{array}$} \\
\hline
\end{tabular}




\section{Cuadro II}

ANÁLISIS DE 235 CASOS CON CONDUCTA SUICIDA Y 470 CONTROLES PAREADOS POR EDAD Y SEXO, EN RELACIÓN CON VIOLENCIA DOMÉSTICA Y A LAS POSIBLES VARIABLES CONFUSORAS, CON ESTIMACIÓN DE LA RM de Mantell- Haenzel para dos controles por caso. Colima, México, julio de 2009

$\begin{array}{ccccc}\text { Casos } & \text { Controles } & \text { Valor de } \chi^{2} & \text { Valor } p & \text { RM de } M-H \\ (n=235) & (n=470) & \text { (IC 95\%) }\end{array}$

\begin{tabular}{|c|c|c|c|c|c|}
\hline \multicolumn{6}{|l|}{ Violencia física } \\
\hline Positivo & 55 & 24 & 47.1 & $<0.0001$ & $5.47(4.11-7.28)$ \\
\hline Negativo & 180 & 446 & & & \\
\hline \multicolumn{6}{|l|}{ Violencia verbal } \\
\hline Positivo & 128 & 45 & 79.1 & $<0.0001$ & $9.28(7.5-10.9)$ \\
\hline Negativo & 107 & 425 & & & \\
\hline \multicolumn{6}{|l|}{ Abuso sexual } \\
\hline Positivo & 13 & 1 & $20.1^{*}$ & $<0.00001$ & $27.46(3.5-211.2)$ \\
\hline Negativo & 222 & 469 & & & \\
\hline \multicolumn{6}{|c|}{ Nivel socioeconómico } \\
\hline Bajo & 45 & 69 & 1.9 & 0.15 & $1.39(0.34-5.62)$ \\
\hline Medio-alto & 190 & 401 & & & \\
\hline \multicolumn{6}{|l|}{ Nivel escolar } \\
\hline Bachillerato & 193 & 371 & 0.19 & 0.35 & $1.21(0.81-1.8)$ \\
\hline Profesional & 42 & 99 & & & \\
\hline \multicolumn{6}{|l|}{ Tabaquismo } \\
\hline Positivo & 40 & 23 & 24.8 & $<0.0001$ & $3.6(2.42-5.32)$ \\
\hline Negativo & 195 & 447 & & & \\
\hline \multicolumn{6}{|l|}{ Uso de drogas $\ddagger$} \\
\hline Positivo & 12 & 3 & $14.1^{*}$ & 0.0002 & $8.4 I(2.3-30.1)$ \\
\hline Negativo & 220 & 467 & & & \\
\hline
\end{tabular}

Cuadro III

ANÁLISIS DE LA INTERACCIÓN ENTRE LAS VARIABLES SELECCIONADAS POR EL MÉTODO RETRÓGRADO EN UN MODELO DE REGRESIÓN LOGÍSTICA MULTIVARIADA*

Variable Coeficiente Valor p RM (IC 95\%)

\begin{tabular}{lcccc} 
Violencia física & 0.24 & 0.46 & 1.27 & $(0.66-2.44)$ \\
\hline Violencia verbal & 2.29 & $<0.000 \mathrm{I}$ & 9.92 & $(6.35-15.37)$ \\
\hline Abuso sexual & 2.88 & 0.008 & 17.85 & $(2.1-152.6)$ \\
\hline Tabaquismo & 0.97 & 0.0037 & 2.64 & $(1.37-5.1)$ \\
\hline Uso de drogas & 0.8 & 0.29 & 2.23 & $(0.5-9.96)$
\end{tabular}

* 702 individuos completaron todas las respuestas. Valor de $\chi^{2}$ para el modelo $=194.3, p<0.0001$

\section{Discusión}

La frecuencia de ideación suicida y de intento suicida resulta muy elevada en el grupo total de estudiantes (15.8 y $7.3 \%$ respectivamente) y coincide con lo encontrado en China ${ }^{13}$, Uganda ${ }^{5}$ y México. ${ }^{12}$ Ambas conductas se mostraron con mayor frecuencia en el sexo femenino, tal como ha sido previamente reportado. ${ }^{13,27}$ Aunque los varones son quienes culminan el suicidio con mayor frecuencia, las mujeres presentan mayor incidencia de ideación e intento suicida. ${ }^{10,28}$ Esta tendencia a la ideación suicida en las mujeres varía de una comunidad a otra, seguramente en relación con patrones culturales peculiares de cada sociedad. ${ }^{13,29,30}$

En ese mismo sentido, nuestro estudio hace manifiesto que las mujeres refirieron significativamente 
mayor violencia física, verbal y sexual, lo cual coincide con el señalamiento de que la violencia doméstica se ejerce predominantemente hacia la mujer en casi todas las sociedades. ${ }^{22,31}$ Mientras que en ellas se observó una menor tendencia hacia el uso de tabaco, alcohol y otras drogas, lo que podría explicarse igualmente por la persistencia de presiones sociales relacionadas con roles de género (por ejemplo: "fumar y usar drogas son conductas poco femeninas").

Una vez ajustado el efecto de sexo y edad, el análisis de casos y controles mostró una fuerte asociación entre la conducta suicida y la percepción de violencia doméstica en todas sus formas, particularmente con el abuso sexual, que arrojó los valores de RM más elevados. Estos hallazgos coinciden con lo reportado previamente, en cuanto a la asociación entre abuso sexual y conducta suicida. ${ }^{16,19}$ Esta asociación entre abuso sexual en casa y la conducta suicida, aunque no se especifique al agresor, obliga a poner mucha mayor atención a la vigilancia de esta conducta delictiva, que bien podría catalogarse como "daños a la salud". Con respecto a la violencia física, los hallazgos actuales indican que esta forma de violencia puede ocasionar también conducta suicida y, eventualmente, consumación de suicidio.

Por otro lado, el papel de la violencia verbal ha sido muy pocas veces considerado dentro de las causantes de psicopatología, al menos desde el enfoque epidemiológico, y ello ha llevado a subestimar este problema, que en algunos sitios es considerado como parte de los controles parentales o estilos de educación "normales" y tal vez por esa razón no ha recibido suficiente atención dentro de los programas orientados a reducir la violencia, los cuales habitualmente se enfocan a la violencia física y al abuso sexual. ${ }^{18,32}$ Cabe destacar que de acuerdo con el análisis multivariado en nuestro estudio, la presencia de violencia verbal se asocia a conducta suicida de forma independiente a la presencia de violencia física y/o sexual, lo cual sugiere que la percepción de agresión verbal en el ámbito doméstico puede llegar a tener tanto o mayor impacto sobre el desarrollo de tendencias autodestructivas en los menores que la violencia física o sexual, aunque el número tan pequeño de estos últimos casos hace difícil establecer su verdadero peso como factores de riesgo independientes. Es muy posible que el maltrato verbal, ya sea en forma de ofensas, descalificaciones, inducción de culpa, o de críticas hirientes, por sí solo incida directamente en una baja autoestima de los menores y ésta pudiera derivar en conductas autodestructivas. ${ }^{32}$ En este sentido, se ha señalado que las relaciones conflictivas entre padres e hijos conducen a ideación e intento suicida. ${ }^{33}$

El estudio también reveló una asociación significativa de la conducta suicida con el uso de tabaco y de drogas. Esta asociación se mantuvo en niveles considerables aún después de su ajuste para las otras variables con la regresión multivariada, por lo que pudieran considerarse factores de riesgo independientes, a pesar del escaso número de respondedores en el caso de drogas. Esto coincide con estudios previos que sugieren una relación directa entre tabaquismo y conducta suicida y en los que el consumo de tabaco parece preceder a esta conducta. 8,34

En cuanto al nivel socioeconómico bajo y al nivel escolar de bachillerato, ambos mostraron cierta asociación positiva con la conducta suicida, tal como se ha encontrado en encuestas previas, ${ }^{13,35}$ sin embargo, en nuestro caso esta asociación no alcanzó niveles significativos y debido a su bajo peso en relación con los otros factores, ambas fueron eliminadas en el modelo multivariado.

El presente estudio tiene las siguientes limitaciones: a) El tipo de población no representa la población total de adolescentes; b) Existe limitante en la confiabilidad de los datos en este tipo de autorrespuesta, particularmente tratándose de temas confidenciales como los aquí señalados, aunque ellas sirven para identificar tendencias; c) No se especifica la gravedad del intento suicida; d) No se incluyeron variables que pudieran ser relevantes como la integración familiar. Sin embargo, los hallazgos permiten hacer las siguientes conclusiones: la ideación suicida y el intento suicida son un problema relevante de salud pública entre los adolescentes universitarios de nuestro país. Su frecuencia es mayor en las mujeres y se asocia significativamente a la violencia doméstica, particularmente al abuso sexual, pero también al maltrato físico y verbal. Esta última es una forma de violencia poco atendida y que parece tener implicaciones graves en la salud mental de los jóvenes. La conducta suicida también se asocia al uso de tabaco y de otras drogas. Por todo lo anterior, se sugiere poner especial atención a las mujeres adolescentes con antecedentes de violencia doméstica, especialmente si ha existido abuso sexual, así como consumo de tabaco o drogas, ya que se trata de una población muy vulnerable hacia la conducta suicida. Por otra parte, deberán implementarse programas preventivos en los que se involucre a padres de familia para buscar estilos de crianza libres de cualquier forma de violencia en un marco de sana disciplina.

\section{Agradecimientos}

Este proyecto fue financiado por la Subsecretaría de Educación Superior e Investigación Científica (SESIC) de la SEP a través del programa de fortalecimiento institucional (PIFI 3.2). 


\section{Referencias}

I. Organización Mundial de la Salud. Informe sobre la Salud en el mundo 200I. Salud Mental, nuevos conocimientos, nuevas esperanzas. Ginebra, Suiza:WHO, 200I: 29-30.

2. Pelkonen M, Marttunen M. Child and adolescent suicide: epidemiology, risk factors, and approaches to prevention. Paediatr Drugs 2003;5: 243- 265. 3. Organización Panamericana de la Salud. Salud de los adolescentes. Washington, DC: OPS/OMS, 1995.

4. Gould MS, Greenberg T,Velting D, Shaffer D. Youth suicide risk and preventive interventions: a review of the past 10 years. J Am Acad Child Adolesc Psychiatry 2003; 42: 386-405.

5. Rudatsikira E, Muula AS, Siziya S, Twa- Twa J. Suicidal ideation and associated factors among school-going adolescents in rural Uganda. BMC Psych 2007; 7: 67-67.

6. Bioulac S, Bourgeois M, Ekouevi DK, Bonnin JM, Gonzales B, Castello MF. Predictive factors of suicide? an 8-year-long prospective longitudinal study of 200 psychiatric inpatients. Encephale 2000;26:I-7.

7. Gerson J, Stanley B. Suicidal and self-injurious behavior in personality disorder: controversies and treatment directions. Curr Psychiatry Rep 2002;4:30- 38.

8. Wilhelm K, Wedgwood L, Niven H, Kay-Lambkin F. Smoking cessation and depression: current knowledge and future directions. Drug Alcohol Rev 2006;25: 97-107.

9. Borges G,Walters EE, Kessler RC.Associations of substance use, abuse, and dependence with subsequent suicidal behavior.Am J Epidemiol 2000;15।:78I-789.

I0. Suominen K, Isometsä E, Suokas J, Haukka J,Achte K, Lönnqvist J. Completed suicide after a suicide attempt: a 37-year follow-up study.Am J Psychiatry 2004; 161: 562- 563.

II. Laurent A, Foussard N, David M, Boucharlat J, Bost M.A 5-year followup study of suicide attempts among French adolescents. J Adolesc Health 1998;22:424- 430.

12. González-Forteza C, Berenzon-Gorn S, Tello-Granados AM, FacioFlores D, Medina-Mora Icaza ME. Ideación suicida y características asociadas en mujeres adolescentes. Salud Publica Mex 1998;40: 430- 437. 13. Liu X, Tein JY, Zhao Z, Sandler IN. Suicidality and correlates among rural adolescents of China. J Adolesc Health 2005;37: 443- 45 I. 14. Roberts RE, Roberts CR, Chen YR. Suicidal thinking among adolescents with a history of attempted suicide.J Am Acad Child Adolesc Psychiatry 1998;37: 1294-1300.

15. Vincent V, Fischer W, Pahud AL, Pascual T, Ladame F. Multiple suicide attempts in adolescence, sexual abuse during childhood and borderline personality disorder. Eur Psychiatry 1997; 12: 189s-189s.

16. Evans E, Hawton K, Rodham K. Suicidal phenomena and abuse in adolescents: a review of epidemiological studies. Child Abuse Negl 2005;29: 45-58
17. Rosenberg ML, Butchart A, Mercy J, Narasimhan V, Waters H, Marshall MS. Interpersonal violence. In: Disease control priorities in developing countries. New York: Oxford University Press and World Bank Group, 2005:755- 770.

18. Páramo CD, Chávez-Hernández AM. Maltrato y suicidio infantil en el estado de Guanajuato. Salud Mental 2007; 30: 59-67

19. Ramos-Lira L, Saltijeral-Méndez MT, Romero-Mendoza M, CaballeroGutiérrez MA, Martínez-Vélez NA.Violencia sexual y problemas asociados en una muestra de usuarias de un centro de salud. Salud Publica Mex 2001; 43:182- 191 .

20. INEGI.Anuario estadístico del Estado de Colima, Instituto Nacional de Geografía e Informática, 2005. Colima, México: INEGI, 2005

21. López J], González de Cossío OM. Examen Médico Automatizado, ISBN 970-32-1770-2. Libro electrónico DGSM-UNAM. México: Programa Universitario de Investigación en Salud (PUIS), 2002.

22. Instituto Nacional de Estadística, Geografía e Informática. Encuesta sobre violencia familiar, 1999. México: INEGI, 2000.

23. The WHO MONICA project. Daily cigarette smoking. Edit by HT Pedoe. WHO Lib Cat, 2003. Geneve, Switz:WHO, 194.

24. Babor TF, De la Fuente JR, Saunders J, Higgins-Biddle, JC.AUDIT the alcohol use disorders identification test: Guidelines for use in primary health care. Geneve:World Health Organization, Division of Mental Health 1989;5: 12- 15.

25. Katz HM. Multivariable Analysis: A Practical Guide for Clinicians. Cambridge: Cambridge University Press NYC, 2000.

26. Pezzullo JC, Sullivan KM. Logistic Regression. [Consultado 2009 febrero 14]. Disponible en:http://statpages.org/logistic.html..

27. Laghi F, Baiocco R, D'Alessio M, Gurrieri G. Suicidal ideation and time perspective in high school students. Eur Psychiatry 2009;24:41- 46. 28. Canetto SS, Sakinofsky I.The gender paradox in suicide. Suicide Life Threat Behav 1998;28: I-23

29. Canetto SS. Women and suicidal behavior: a cultural analysis. Am J Orthopsychiatry 2008;78(2):259- 266.

30. Hankin BL, Mermelstein R, Roesch L. Sex differences in adolescent depression: stress exposure and reactivity models. Child Dev 2007;78(I):279-295.

31. García-Moreno C, OPS.Violencia contra la mujer: género y equidad en la salud. Washington, DC: OPS, 2000:7-38.

32. Sachs-Ericsson N, Verona E, Joiner T, Preacher KJ. Parental verbal abuse and the mediating role of self-criticism in adult internalizing disorders. J Affect Disord 2006;93:71-78.

33. Palacios-Delgado JR,Andrade-Palos P. Influencia de las prácticas parentales en las conductas problema en adolescentes. Investigación Universitaria Multidisciplinaria 2008;7:7-18.

34. Bronisch T, Höfler M, Lieb R. Smoking predicts suicidality: findings from a prospective community study.J Affect Disord 2008; I08: I35-I 45.

35. Cheung AH, Dewa CS. Canadian community health survey: major depressive disorder and suicidality in adolescents. Health Policy 2006;2:76-89. 